\title{
Effective Dose of Lactosucrose on Fecal Flora and Fecal Metabolites of Humans
}

\author{
Hiroyoshi Hara, ${ }^{1}$ Shou-Tou Li, ${ }^{1}$ Masakuni SAsaki, ${ }^{1}$ \\ Tsukasa Maruyama, ${ }^{1}$ Atsushi Terada, ${ }^{1}$ Yukiko Ogata, ${ }^{2}$ \\ Koki Fujita, ${ }^{2}$ Hiroshi Ishigami, ${ }^{2}$ Kozo Hara, ${ }^{2}$ \\ Isao FujIMORI ${ }^{3}$ and Tomotari Mitsuoka ${ }^{1}$ \\ ${ }^{1}$ Department of Food Hygiene, Nippon Veterinary and Animal Science University, \\ 1-7-1 Kyonan-cho, Musashino, Tokyo 180, \\ ${ }^{2}$ Ensuiko Sugar Refining Co., Ltd, 1-4-21 Yaesu, Chuo-ku, Tokyo 103, and \\ ${ }^{3}$ Taiyo Pet Food Co., Ltd., Tokyo Kaijo Bldg. 2-3-3 Shiba, Minato-ku, Tokyo 105
}

(Received for publication, June 2, 1994)

\begin{abstract}
Lactosucrose ( $4^{G_{-}} \beta$-D-galactosylsucrose) was fermented in vitro by bifidobacteria and lactobacilli, and to a limited degree by the Bacteroides fragilis group, clostridia, eubacteria, and enterobacteriaceae. The effects of dietary lactosucrose on the fecal flora and fecal metabolites were studied in eight healthy volunteers (20-23 years of age) who ingested $3 \mathrm{~g}$ of lactosucrose/day for 7 days followed by $6 \mathrm{~g}$ of lactosucrose/day for 7 consecutive days. During lactosucrose intake, the counts of bifidobacteria were increased significantly $(p<0.001)$, whereas the counts of clostridia, including Clostridium perfringens, and bacteroidaceae were decreased significantly $(p<0.05)$ compared with the values before the intake. The total bacterial counts were decreased significantly $(p<0.05)$ on day 14 during the intake. The frequency of occurrence of lecithinase-negative clostridia was decreased significantly $(p<0.05)$ when compared with the values before and after the intake. No detectable changes occurred in the counts of other organisms throughout the experimental periods. Fecal concentrations of ammonia, sulfide, phenol, ethylphenol, skatol and indole were decreased significantly $(p<0.05)$ during lactosucrose intake. Acetic acid and lactic acid were increased significantly $(p<0.05)$ during the intake. Fecal enzyme activity of $\beta$-glucuronidase was decreased significantly $(p<0.05)$ on day 14 of the intake. Serum very low density lipoprotein (VLDL) was increased significantly $(p<0.01)$ on day 14 during the intake. Mean fecal $\mathrm{pH}$ values decreased from 6.3 to 5.9, and mean water content increased $3.6 \%$ during the intake. Fecal weight was increased slightly during the intake. The results obtained showed that the effective dose of lactosucrose for all healthy adults is $3 \mathrm{~g} /$ day.
\end{abstract}

Key words: enzyme activity; intestinal flora; lactosucrose; organic acids; putrefactive products; serum lipids 
The intestinal flora is composed of 100 trillion bacteria including 100 species, and contains a variety of enzymes that perform extremely varied types of metabolism in the intestine. They not only contribute to normal physiological functions, but also participate significantly in the causation of various disease states by biotransforming a variety of ingested or endogenous compounds to beneficial or harmful derivatives. Thus, this transformation may influence drug efficacy, toxicity, carcinogenesis and aging (17).

Recently, it has been reported that lactosucrose [ $4^{\mathrm{G}}-\beta$-D-galactosylsucrose or $O$ - $\beta$-D-galacto-pyranosyl- $(1 \rightarrow 4)-O$ - $\alpha$-D-glucopyranosyl- $(1 \leftarrow 2)-\beta$-D-fructofuranoside] selectively enhanced the growth of bifidobacteria in the human intestine $(21,34)$ and inhibited intestinal clostridia of dogs and cats, resulting in the reduction of putrefactive products in the intestine $(30,31)$.

In the present study, we examined the effects of lactosucrose on the fecal flora, weight, water content, $\mathrm{pH}$, metabolic activities, and the serum lipid levels in eight healthy volunteers.

\section{MATERIALS AND METHODS}

Preparation and composition of lactosucrose. Lactosucrose (LS) was prepared from lactose and sucrose by $\beta$-fructofuranosidase from Arthrobacter sp. K-1(5). The LS preparation was composed of $95.1 \%$ lactosucrose, $2.8 \%$ sucrose, $0.4 \%$ lactose, and $1.7 \%$ other oligosaccharides.

Fermentation of $L S$ in vitro. A total of 103 strains of human intestinal bacteria including 22 strains of six species of bifidobacteria were used in this test. The saccharides tested were LS (Ensuiko Sugar Refining Co., Ltd., Tokyo), glucose (Wako Pure Chemical Co., Ltd., Tokyo), raffinose (Wako), lactulose (Sigma), fructo-oligosaccharides (FOS, Meiji Seika Co., Ltd., Tokyo), and soybean oligosaccharides refined (SOR, Calpis Food Industry Co., Ltd., Tokyo).

One milliliter of peptone yeast extract Fildes solution (PYF) broth with $0.5 \%$ of the saccharides were inoculated with $0.03 \mathrm{ml}\left(10^{8} \mathrm{CFU} / \mathrm{ml}\right)$ of the test organisms using an automatic multipoint inoculator 120 (Lifetec Co., Ltd., Saitama). The organisms were pre-cultured in PYF broth with $1 \%$ glucose, centrifuged at 3,000 $\mathrm{rpm}$ at $4^{\circ} \mathrm{C}$ for $20 \mathrm{~min}$, and suspended at $0.5 \mathrm{ml}$ with a diluent solution composed of $0.1 \%$ sodium thioglycolate, $0.1 \% \mathrm{~L}$-cysteine $\mathrm{HCl}$ and $0.85 \% \mathrm{NaCl}$. The inoculated media were incubated anaerobically at $37^{\circ} \mathrm{C}$ for $96 \mathrm{hr}$ by the steel wool method. After incubation, the $\mathrm{pH}$ of each medium was measured using an automatic multipoint $\mathrm{pH}$ meter 5051 (Lifetec Co., Ltd.), and scored in the following manner: $\mathrm{pH}>6.0 ;(+), \mathrm{pH} 5.5-5.9 ;+, \mathrm{pH} 5.0-5.4 ;+\mathrm{H}, \mathrm{pH}<4.9$.

Subjects and diet. The subjects were eight healthy male volunteer students (Department of Food Hygiene, Nippon Veterinary and Animal Science University, Tokyo) who ranged in age from 20 to 23 years. They consumed the normal freechoice diet for 2 weeks before (CNT-1) and after (CNT-2) the LS period, as shown in Table 1. They were given the normal diet plus a cup of coffee with $3 \mathrm{~g} /$ day (LS-1) and $6 \mathrm{~g} /$ day (LS-2) of LS for the first and second weeks, respectively. None 
Table 1. Experimental schedule

\begin{tabular}{|c|c|c|c|c|}
\hline \multirow{2}{*}{$\begin{array}{l}\begin{array}{l}\text { Diet ingested } \\
\text { Experimental period }\end{array} \\
\text { Weeks }\end{array}$} & \multirow{2}{*}{$\begin{array}{c}\text { Control diet } \\
\leftarrow \text { CNT 1 } \rightarrow \\
\text { Before intake } \\
\qquad \leftarrow 2 \rightarrow\end{array}$} & $\leftarrow \underset{\text { Luring }}{\stackrel{\text { Lactc }}{\rightarrow}}$ & $\begin{array}{l}\text { crose } \\
\stackrel{\mathrm{LS}}{\leftarrow}-2 \rightarrow \\
\text { ntake }\end{array}$ & \multirow{2}{*}{ 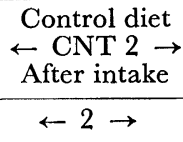 } \\
\hline & & $\leftarrow 1 \rightarrow$ & $\leftarrow 1 \rightarrow$ & \\
\hline Lactosucrose (g/day) & & 3 & 6 & \\
\hline Sampling day & 0 & 7 & 14 & 14 \\
\hline \multicolumn{5}{|l|}{ Item for analysis; } \\
\hline Moisture content (\%) & * & $*$ & $*$ & $*$ \\
\hline $\mathrm{pH}$ & $*$ & $*$ & $*$ & $*$ \\
\hline Fecal weight (g) & $*$ & $*$ & $*$ & $*$ \\
\hline Fecal flora & $*$ & $*$ & $*$ & $*$ \\
\hline Metabolic products & $*$ & $*$ & $*$ & $*$ \\
\hline Enzyme activity & $*$ & $*$ & $*$ & $*$ \\
\hline Serum lipids & $*$ & $*$ & $*$ & $*$ \\
\hline
\end{tabular}

*: Items examined.

Table 2. Media and cultural methods for comprehensive investigation of intestinal flora

\begin{tabular}{|c|c|c|c|}
\hline Medium & $\begin{array}{l}\text { Organisms } \\
\text { enumerated }\end{array}$ & $\begin{array}{l}\text { Incubation method } \\
\text { and dilution }\end{array}$ & $\begin{array}{l}\text { Incubation } \\
\text { at } 37^{\circ} \mathrm{C}\end{array}$ \\
\hline \multicolumn{4}{|l|}{ Non-selective media } \\
\hline $\begin{array}{l}\text { Medium } 10 \\
\text { (modified) }\end{array}$ & $\begin{array}{l}\text { Fastidious } \\
\text { anaerobes }\end{array}$ & $\begin{array}{l}\text { Plate-in-bottle method } \\
10^{-7}, 10^{-8}\end{array}$ & 4 days \\
\hline EG agar & Anaerobes & \multirow{2}{*}{$\begin{array}{l}\text { Steel wool method } \\
\text { replaced air with } \mathrm{CO}_{2} \\
10^{-6}, 10^{-7}, 10^{-8}\end{array}$} & \multirow{2}{*}{\} 3 days } \\
\hline BL agar & Anaerobes & & \\
\hline Trypticase soy & Aerobes & Air & \multirow[t]{3}{*}{ 1-2 days } \\
\hline blood agar & & $10^{-5}, 10^{-6}, 10^{-7}$ & \\
\hline Selective media & & $10^{-1}, 10^{-3}, 10^{-5}, 10^{-7}$ & \\
\hline BS agar & Bifidobacteria & \multirow{5}{*}{$\begin{array}{l}\text { Steel wool method } \\
\quad \text { replaced air with } \mathrm{CO}_{2}\end{array}$} & \multirow{5}{*}{3 days } \\
\hline ES agar & Eubacteria & & \\
\hline NBGT agar & Bacteroidaceae & & \\
\hline Neomycin Nagler agar & Clostridia & & \\
\hline VS agar & $\begin{array}{l}\text { Veillonellae and } \\
\text { Peptostreptococci }\end{array}$ & & \\
\hline LBS agar (modified) & Lactobacilli & Replaced air with $\mathrm{CO}_{2}$ & 2 days \\
\hline DHL agar & Enterobacteriaceae & \multirow{5}{*}{ Air } & 1 day \\
\hline TATAC agar & Streptococci & & \multirow{4}{*}{2 days } \\
\hline PEES agar & Staphylococci & & \\
\hline NAC agar & Pseudomonas & & \\
\hline Potato dextrose agar & Yeasts and molds & & \\
\hline Media after heat treatment & & $10^{-1}, 10^{-3}, 10^{-5}$ & \\
\hline BL agar and CW agar & Clostridia & $\begin{array}{l}\text { Steel wool method } \\
\text { replaced air with } \mathrm{CO}_{2}\end{array}$ & 2 days \\
\hline
\end{tabular}

of the subjects were given antibiotic treatment or other therapy or foods with abundant viable cultures for 1 month prior to and during the experiments.

This work was performed in accordance with the Helsinki Declaration as updated in Tokyo, 1975. 
Table 3. Utilization of six oligosaccharides by various intestinal bacteria

\begin{tabular}{|c|c|c|c|c|c|c|c|c|}
\hline Organism & $\begin{array}{l}\text { No. of } \\
\text { strains }\end{array}$ & control & Glucose & $\begin{array}{l}\text { Lacto- } \\
\text { sucrose }\end{array}$ & Lactulose & FOS & SOR & Raffinose \\
\hline \multicolumn{9}{|l|}{ Bifidobacterium } \\
\hline B. longum & 6 & 一 & $H$ & H & H & H & $H$ & H \\
\hline B. adolescentis & 7 & 一 & H & H & $H$ & H & H & H \\
\hline B. breve & 3 & - & H & $H$ & $H$ & H & Ht & H \\
\hline B. bifidum & 3 & - & $H$ & + & $H$ & - & - & - \\
\hline B. infantis & 2 & - & H & + & H & + & + & + \\
\hline B. pseudocatenulatum & 1 & - & H & Ht & H & H & H & + \\
\hline \multicolumn{9}{|l|}{ Lactobacillus } \\
\hline L. acidophilus & 3 & - & H & + & + & H & + & $(+)$ \\
\hline L. salivarius & 2 & 一 & H & $H$ & $H$ & H & H & $H$ \\
\hline L. casei & 2 & 一 & + & H & $H$ & - & - & - \\
\hline \multicolumn{9}{|l|}{ Bacteroides } \\
\hline B. vulgatus & 12 & - & H & + & + & + & $(+)$ & $(+)$ \\
\hline B. distasonis & 5 & - & H & + & + & + & + & + \\
\hline B. fragilis & 3 & - & $H$ & + & + & H & + & H \\
\hline B. thetaiotamicron & 2 & 一 & $H$ & + & + & + & + & + \\
\hline B. uniformis & 1 & - & H & $H$ & + & $H$ & + & $H$ \\
\hline B. melaninogenicus & 1 & - & + & - & - & - & - & - \\
\hline \multicolumn{9}{|l|}{ Rikenella } \\
\hline R. microfusus & 1 & - & H & + & + & + & $(+)$ & $(+)$ \\
\hline \multicolumn{9}{|l|}{ Clostridium } \\
\hline C. perfringens & 6 & - & H & $(+)$ & + & $(+)$ & $(+)$ & $(+)$ \\
\hline C. bifermentans & 2 & - & + & + & $(+)$ & $(+)$ & - & - \\
\hline C. paraputrificum & 3 & 一 & H & $(+)$ & + & - & - & - \\
\hline C. ramosum & 2 & - & $H$ & + & + & + & $(+)$ & $(+)$ \\
\hline C. butyricum & 1 & - & H & + & H & + & + & + \\
\hline Clostridium spp. & 4 & - & $H$ & + & + & $(+)$ & $(+)$ & - \\
\hline \multicolumn{9}{|l|}{ Eubacterium } \\
\hline E. aerofaciens & 8 & - & $H$ & $(+)$ & + & + & + & - \\
\hline E. limosum & 2 & - & H & - & - & - & - & - \\
\hline E. lentum & 1 & - & - & - & - & - & - & - \\
\hline Eubacterium spp. & 1 & - & H & + & + & - & - & - \\
\hline \multicolumn{9}{|l|}{ Peptostreptococcus } \\
\hline$P$. parvulus & 1 & - & H & - & - & - & - & - \\
\hline \multicolumn{9}{|l|}{ Escherichia } \\
\hline E. coli & 3 & - & + & $(+)$ & $(+)$ & - & - & - \\
\hline \multicolumn{9}{|l|}{ Salmonella } \\
\hline S. enteritidis & 1 & 一 & H & $(+)$ & - & - & - & $(+)$ \\
\hline S. typhimurium & 1 & - & H & $(+)$ & $(+)$ & - & - & $(+)$ \\
\hline \multicolumn{9}{|l|}{ Proteus } \\
\hline P. mirabilis & 1 & - & $H$ & $(+)$ & $(+)$ & $(+)$ & $(+)$ & $(+)$ \\
\hline P. vulgaris & 1 & - & $H$ & + & + & $(+)$ & $(+)$ & - \\
\hline \multicolumn{9}{|l|}{ Aeromonas } \\
\hline A. hydrophyla & 2 & - & $H$ & - & - & $(+)$ & - & - \\
\hline A. sobria & 1 & - & + & - & - & $(+)$ & - & - \\
\hline \multicolumn{9}{|l|}{ Enterococcus } \\
\hline E. faecalis & 2 & 一 & H & + & + & $(+)$ & $(+)$ & $(+)$ \\
\hline \multicolumn{9}{|l|}{ Staphylococcus } \\
\hline S. aureus & 4 & - & $H$ & + & + & $(+)$ & $(+)$ & $(+)$ \\
\hline
\end{tabular}




\begin{tabular}{|c|c|c|c|c|c|c|c|c|}
\hline$B$ cereus & 1 & 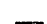 & $H$ & 1 & 1 & 1 & 1 & + \\
\hline B. mycoides & 1 & - & $H$ & + & + & + & + & + \\
\hline
\end{tabular}

FOS, fructo-oligosaccharides; SOR, soybean oligosaccharides refined. Judgment of bacterial growth: - $\mathrm{pH} \geqq 6.0,(+) ; 5.5 \leqq \mathrm{pH}<5.9,+; 5.0 \leqq \mathrm{pH}<5.4$, $\mathrm{H} ; \mathrm{pH}<4.9$

Sampling of feces and blood. Freshly voided fecal samples were collected from each subject on immediately before lactosucrose intake, on days 7 and 14 during the intake, and on day 14 after the intake. The samples were weighed to determine fecal output and were stored immediately at $4^{\circ} \mathrm{C}$. Fecal flora, water content and enzyme activities were analyzed within 3 hours after the collection of samples. The remainder of the samples were frozen at $-80^{\circ} \mathrm{C}$ for later analysis of bacterial metabolites.

Blood samples were obtained $60 \mathrm{~min}$ before and after breakfast on the same day of collection of fecal specimens.

Bacteriological analysis. The fecal flora was analyzed using the method and media of Mitsuoka et al. (18) as shown in Table 2. Two grams of feces were suspended in $18 \mathrm{ml}$ of anaerobic diluent and mixed thoroughly, then a decimal dilution series $\left(10^{-1}-10^{-8}\right)$ was prepared. From appropriate dilutions, $0.05-\mathrm{ml}$ aliquots were inoculated to 4 non-selective and 11 selective media. In addition, dilutions $\left(10^{-1}\right.$ $10^{-5}$ ) of the fecal specimens were heated at $80^{\circ} \mathrm{C}$ for $10 \mathrm{~min}$ to select for clostridial spores and a portion of $0.1 \mathrm{ml}$ of each dilution was inoculated onto $\mathrm{BL}$ agar and CW agar (Nissui Seiyaku, Co., Ltd., Tokyo). After incubation, each plate was examined for bacterial colonies. The identification of 16 bacterial groups, yeasts and molds was performed with Gram-reaction, colonial and cellular morphologies, spore formation, aerobic growth, and selected biochemical characteristics. The bacterial counts are expressed as the $\log _{10}$ of the number of bacteria per gram wet feces.

Analysis of fecal metabolites. Measurement of fecal ammonia was carried out using a potentiometer IOL-30 (DKK Co., Ltd., Tokyo) with ammonia gas sensing electrode 1716 using the method of Terada et al. (32). For sulfide analysis, $1 \mathrm{~g}$ of feces was dissolved in $44 \mathrm{ml}$ of distilled water, to which was added $5 \mathrm{ml}$ of S-DIMA ( $40 \mathrm{~g} \mathrm{NaOH}, 10 \mathrm{~g}$ L-ascorbic acid, $9.3 \mathrm{~g}$ EDTA $2 \mathrm{Na}, 500 \mathrm{ml}$ glycerine, and $485.7 \mathrm{ml}$ distilled water), and then measured using a sulfide electrode 7100 (DKK Co., Ltd.). The concentrations of fecal organic acids were determined by highperformance liquid chromatography organic acid analysis system (HPLCOA, Shimazu Seisakusho Co., Ltd., Tokyo). One gram of fecal samples was homogenized with $5 \mathrm{ml}$ of distilled water and centrifuged at $3,000 \mathrm{rpm}$ at $4^{\circ} \mathrm{C}$ for $20 \mathrm{~min}$. A $10-\mathrm{ml}$ portion of the suspension was analyzed on HPLCOA with a two-column serial connection of SCR-102H (8 mm I.D. $\times 300 \mathrm{~mm} \mathrm{~L}$.) added a guard column SCR-102H (6 mm I.D. $\times 50 \mathrm{~mm} \mathrm{L.).} \mathrm{Operating} \mathrm{parameters} \mathrm{were} \mathrm{as} \mathrm{follows:}$ column oven temperature, $40^{\circ} \mathrm{C}$; flow rate of mobile phase $(5 \mathrm{mM} p$-toluenesulfonic acid aqueous solution), $0.8 \mathrm{ml} / \mathrm{min}$; flow rate of buffer $(20 \mathrm{mM}$ Bis-Tris aqueous 
solution containing $5 \mathrm{mM}$ p-toluenesulfonic acid and $100 \mathrm{mM}$ EDTA), $0.8 \mathrm{ml} /$ min. Fecal contents of indole, skatol and phenols were analyzed by gas chromatography using the method of Yoshihara (35). Fecal enzyme activities were determined using the method of Rowland et al. (24).

Assay of serum lipids. Serum phospholipids (27), triglycerides (16), free fatty acids (26), and cholesterol (13) were measured enzymatically, and lipoprotein was measured by use of a turbidimetric method (25) and agarose gel electrophoresis assay (23).

Measurement of fecal properties. Fecal water contents were measured by using approximately $1 \mathrm{~g}$ samples weighed before and after drying in a vacuum oven 625 (Ikemoto Rika Kogyo Co., Ltd., Tokyo) at $105^{\circ} \mathrm{C}$. Fecal $\mathrm{pH}$ values were determined with a flat glass electrode (DKK Co., Ltd., Tokyo). The weight of fecal output for $24 \mathrm{hr}$ was measured for 3 consecutive days, including the days of sampling for the bacterial analyses.

Statistical analysis of data. Student's $t$-test and the method of Fisher's direct probability calculation were used for statistical analysis of the fecal flora. Student's $t$-test was used for analysis of $\mathrm{pH}$ value, moisture content, weight, enzyme activities and metabolites of feces and serum lipids.

\section{RESULTS}

\section{Utilization of LS In Vitro}

The results of fermentation test in vitro of six saccharides including LS are shown in Table 3. LS was fermented by all the bifidobacteria tested, lactobacilli including $L$. acidophilus, $L$. salivarius and $L$. casei as effectively as glucose, and other bacteria, such as Bacteroides fragilis group, clostridia, Staphylococcus aureus, Enterococcus faecalis, bacilli, Salmonella, and Escherichia coli to a lesser degree than glucose. FOS, SOR and raffinose showed similar fermentation patterns, except that they were not fermented by B. bifidum, L. casei, C. paraputrificum, E. coli, and Salmonella (except for raffinose).

Fecal Flora Analysis

The effects of LS intake on the composition of fecal flora are shown in Table 4. The levels of bifidobacteria were significantly $(p<0.001)$ increased during LS intake, while the levels of lecithinase-positive clostridia including Clostridium perfringens and bacteroidaceae were significantly $(p<0.05)$ decreased during the intake, expect for the levels of $C$. perfringens in one volunteer. The percentage of bifidobacteria to total bacteria were improved from $10.5 \%$ before the intake to $38.9 \%$ and $38.1 \%$ on days 7 and 14 in the LS period, respectively. The levels of total bacteria were significantly $(p<0.05)$ decreased on day 14 in the LS period. In frequency of occurrence, lecithinase-negative clostridia during LS intake were decreased significantly $(p<0.05)$ when compared with before and after the intake. No detectable changes occurred in the levels of other organisms throughout the experimental period. 
Table 4. Effect of lactosucrose intake on fecal flora of 8 human volunteers

\begin{tabular}{|c|c|c|c|c|}
\hline \multirow{2}{*}{ Organism } & \multirow{2}{*}{$\begin{array}{l}\text { Before intake } \\
\quad \text { day } 0\end{array}$} & \multicolumn{2}{|c|}{ During intake } & \multirow{2}{*}{$\begin{array}{l}\text { After intake } \\
\text { day } 14\end{array}$} \\
\hline & & day 7 & day 14 & \\
\hline Total bacteria & $11.01 \pm 0.11^{a}$ & $10.94 \pm 0.11$ & $10.87 \pm 0.07 *$ & $10.93 \pm 0.18$ \\
\hline Bifidobacteria & $\begin{array}{c}10.03 \pm 0.15 \\
(100)^{b}\end{array}$ & $\begin{array}{c}10.53 \pm 0.11 * * * \\
(100)\end{array}$ & $\begin{array}{c}10.45 \pm 0.11 \text { *** } \\
(100)\end{array}$ & $\begin{array}{c}9.92 \pm 0.17 \\
(100)\end{array}$ \\
\hline Bacteroidaceae & $\begin{array}{c}10.80 \pm 0.12 \\
(100)\end{array}$ & $\begin{array}{c}10.63 \pm 0.10^{*} \\
(100)\end{array}$ & $\begin{array}{c}10.50 \pm 0.11^{* *} \\
(100)\end{array}$ & $\begin{array}{c}10.72 \pm 0.19 \\
(100)\end{array}$ \\
\hline Eubacteria & $\begin{array}{c}9.95 \pm 0.38 \\
(100)\end{array}$ & $\begin{array}{c}9.57 \pm 0.39 \\
(100)\end{array}$ & $\begin{array}{c}9.58 \pm 0.36 \\
(100)\end{array}$ & $\begin{array}{c}9.64 \pm 0.42 \\
(100)\end{array}$ \\
\hline Peptococcaceae & $\begin{array}{c}9.55 \pm 0.26 \\
(100)\end{array}$ & $\begin{array}{c}9.14 \pm 0.69 \\
(100)\end{array}$ & $\begin{array}{c}9.19 \pm 0.58 \\
(100)\end{array}$ & $\begin{array}{c}9.69 \pm 0.47 \\
(100)\end{array}$ \\
\hline Megasphaerae & $\begin{array}{c}9.08 \pm 0.67 \\
(88)\end{array}$ & $\begin{array}{c}8.94 \pm 0.52 \\
(63)\end{array}$ & $\begin{array}{c}8.60 \pm 0.67 \\
(88)\end{array}$ & $\begin{array}{c}9.46 \pm 0.41 \\
(88)\end{array}$ \\
\hline Curved rods & $\begin{array}{c}9.03 \pm 0.49 \\
(50)\end{array}$ & $\begin{array}{l}8.80 \\
(25)\end{array}$ & $\begin{array}{l}8.60 \\
(25)\end{array}$ & $\begin{array}{c}9.05 \pm 0.48 \\
(50)\end{array}$ \\
\hline Veillonellae & $\begin{array}{c}6.80 \pm 1.21 \\
(50)\end{array}$ & $\begin{array}{l}6.65 \\
(25)\end{array}$ & $\begin{array}{l}7.43 \\
\quad(25)\end{array}$ & $\begin{array}{c}6.79 \pm 0.16 \\
(38)\end{array}$ \\
\hline \multicolumn{4}{|l|}{ Clostridia } & \\
\hline Lecithinase-positive & $\begin{array}{c}5.74 \pm 1.38 \\
(88)\end{array}$ & $\begin{array}{c}3.78 \pm 0.92 * \\
(88)\end{array}$ & $\begin{array}{c}3.62 \pm 0.89 * \\
(88)\end{array}$ & $\begin{array}{c}4.40 \pm 1.39 \\
(100)\end{array}$ \\
\hline Lecithinase-negative & $\begin{array}{c}9.33 \pm 0.35 \\
(100)\end{array}$ & $\begin{array}{c}8.90 \pm 0.52 \\
(50)^{*}\end{array}$ & $\begin{array}{c}8.50 \pm 0.59 \\
(38)^{*}\end{array}$ & $\begin{array}{c}9.26 \pm 0.50 \\
(100)\end{array}$ \\
\hline Lactobacilli & $\begin{array}{c}7.68 \pm 1.78 \\
(100)\end{array}$ & $\begin{array}{c}7.28 \pm 1.16 \\
(100)\end{array}$ & $\begin{array}{c}7.77 \pm 1.08 \\
(100)\end{array}$ & $\begin{array}{c}7.34 \pm 1.55 \\
(100)\end{array}$ \\
\hline Enterobacteriaceae & $\begin{array}{c}8.81 \pm 1.22 \\
(100)\end{array}$ & $\begin{array}{c}8.51 \pm 0.55 \\
(100)\end{array}$ & $\begin{array}{c}8.11 \pm 1.17 \\
(100)\end{array}$ & $\begin{array}{c}8.79 \pm 0.46 \\
(100)\end{array}$ \\
\hline Pseudomonas & $\begin{array}{c}3.41 \pm 0.85 \\
(63)\end{array}$ & $\begin{array}{c}3.94 \pm 0.53 \\
(38)\end{array}$ & $\begin{array}{c}4.04 \pm 0.75 \\
(38)\end{array}$ & $\begin{array}{c}3.01 \pm 0.32 \\
(88)\end{array}$ \\
\hline Streptococci & $\begin{array}{c}8.43 \pm 0.75 \\
(100)\end{array}$ & $\begin{array}{c}8.53 \pm 1.11 \\
(100)\end{array}$ & $\begin{array}{c}8.56 \pm 0.52 \\
(100)\end{array}$ & $\begin{array}{c}8.61 \pm 0.87 \\
(100)\end{array}$ \\
\hline Staphylococci & $\begin{array}{c}4.70 \pm 1.36 \\
(75)\end{array}$ & $\begin{array}{l}3.62 \\
(25)\end{array}$ & $\begin{array}{l}3.10 \\
(25)\end{array}$ & $\begin{array}{c}3.44 \pm 1.40 \\
(38)\end{array}$ \\
\hline Bacilli & $\begin{array}{c}4.28 \pm 1.66 \\
(63)\end{array}$ & $\begin{array}{c}2.97 \pm 0.94 \\
(38)\end{array}$ & $\begin{array}{l}3.19 \\
(25)\end{array}$ & 2.54 \\
\hline Yeasts & $\begin{array}{c}3.24 \pm 0.52 \\
(63)\end{array}$ & $\begin{array}{c}3.50 \pm 0.55 \\
(63)\end{array}$ & $\begin{array}{c}3.60 \pm 0.96 \\
(63)\end{array}$ & $\begin{array}{c}3.09 \pm 0.55 \\
(75)\end{array}$ \\
\hline Molds & $\begin{array}{l}1.60 \\
(13)\end{array}$ & (0) & $\begin{array}{l}2.30 \\
(13)\end{array}$ & (0) \\
\hline
\end{tabular}

a Data expressed as mean log number per gram feces \pm S.D.

$b$ Figures in parentheses are frequency of occurrence $(\%)$.

Significant difference from the counts of day 0 (before the intake):

$* p<0.05, * * p<0.01, * * * p<0.001$.

\section{Fecal Metabolic Products}

A significant decrease $(p<0.05)$ in the amounts of fecal ammonia, sulfide, indole, phenol, and skatol were observed during LS intake, compared with those before the intake (Table 5).

The concentrations of total organic acids, lactic acid, and acetic acid were significantly $(p<0.05)$ increased during the intake (Table 6$)$. 
Table 5. Effect of lactosucrose intake on fecal ammonia and fecal putrefactive products of 8 human volunteers

\begin{tabular}{lcccr}
\hline \multirow{2}{*}{ Product } & Before intake & \multicolumn{2}{c}{ During intake } & \multirow{2}{*}{$\begin{array}{c}\text { After intake } \\
\text { Day } 14\end{array}$} \\
\cline { 3 - 4 } & \multicolumn{1}{c}{ Day } & Day 7 & Day 14 & \\
\hline Ammonia & $244.8 \pm 139.9 a$ & $96.4 \pm 48.0^{*}$ & $87.3 \pm 46.0^{*}$ & $248.0 \pm 79.8$ \\
Sulfide & $5.1 \pm 1.6$ & $3.3 \pm 1.2^{*}$ & $3.1 \pm 1.4^{*}$ & $4.1 \pm 1.7$ \\
Phenol & $31.1 \pm 19.9$ & $11.4 \pm 3.2^{*}$ & $11.9 \pm 6.5^{*}$ & $21.0 \pm 9.1$ \\
p-Cresol & $54.0 \pm 30.3$ & $37.7 \pm 32.2$ & $35.4 \pm 35.6$ & $48.5 \pm 31.6$ \\
Ethylphenol & $9.5 \pm 9.5$ & $2.1 \pm 2.2$ & $1.8 \pm 2.2$ & $5.5 \pm 5.5$ \\
Indole & $53.8 \pm 23.4$ & $16.4 \pm 10.5 * *$ & $13.6 \pm 8.7 * *$ & $31.8 \pm 18.1$ \\
Skatol & $21.9 \pm 20.7$ & $9.3 \pm 12.9$ & $5.9 \pm 4.9$ & $17.8 \pm 14.6$ \\
\hline
\end{tabular}

a Data expressed as mean $\pm \mathrm{SD}$ of $\mu \mathrm{g} / \mathrm{g}$ wet feces.

Statistically significant at $*=p<0.05$, and $* *=p<0.01$ levels when compared with the values before the intake.

Table 6. Effect of lactosucrose intake on fecal organic acids of 8 human volunteers

\begin{tabular}{lcccc}
\hline \multirow{2}{*}{ Product } & $\begin{array}{c}\text { Before intake } \\
\text { Day 0 }\end{array}$ & Day 7 & Day 14 & $\begin{array}{c}\text { After intake } \\
\text { Day 14 }\end{array}$ \\
\cline { 2 - 4 } & $26.48 \pm 3.39 a$ & $33.70 \pm 7.12^{*}$ & $35.12 \pm 7.23^{*}$ & $28.00 \pm 4.11$ \\
Total organic acids & $2.51 \pm 1.30$ & $2.73 \pm 2.11$ & $2.81 \pm 1.83$ & $2.63 \pm 1.41$ \\
Pyruvic & $1.12 \pm 0.42$ & $2.05 \pm 1.24$ & $2.22 \pm 1.22$ & $1.42 \pm 1.30$ \\
Malic & $2.18 \pm 1.26$ & $3.21 \pm 1.86$ & $3.87 \pm 2.50$ & $2.64 \pm 1.52$ \\
Succinic & $2.62 \pm 0.87$ & $4.08 \pm 2.44^{* *}$ & $4.01 \pm 0.80^{*}$ & $2.81 \pm 1.13$ \\
Lactic & $1.02 \pm 0.68$ & $1.33 \pm 0.52$ & $1.30 \pm 0.51$ & $1.20 \pm 0.80$ \\
Formic & $8.76 \pm 1.03$ & $12.30 \pm 1.22^{*}$ & $11.51 \pm 1.19 *$ & $9.13 \pm 1.12$ \\
Acetic & $3.25 \pm 0.47$ & $4.20 \pm 1.70$ & $4.17 \pm 1.21$ & $3.50 \pm 0.86$ \\
Propionic & $0.20 \pm 0.07$ & $0.15 \pm 0.07$ & $0.42 \pm 0.58$ & $0.17 \pm 0.07$ \\
iso-Butyric & $2.88 \pm 1.15$ & $2.00 \pm 0.88$ & $2.70 \pm 0.99$ & $2.58 \pm 0.66$ \\
Butyric & $0.24 \pm 0.24$ & $0.07 \pm 0.11$ & $0.18 \pm 0.11$ & $0.23 \pm 0.41$ \\
iso-Valeric & $1.70 \pm 1.02$ & $1.58 \pm 1.33$ & $1.93 \pm 1.59$ & $1.69 \pm 0.98$ \\
Valeric & & & &
\end{tabular}

a Data expressed as mean \pm SD of $\mathrm{mg} / \mathrm{g}$ wet feces.

Statistically significant at $*=p<0.05$ and $* *=p<0.01$ levels when compared with the values before the intake.

\section{Fecal Bacterial Enzyme Activities}

Fecal $\beta$-glucuronidase activity was significantly $(p<0.05)$ decreased on day 14 during LS intake compared with before the intake (Table 7). Other enzyme activities showed also a tendency to decrease during LS intake.

Lipid Analysis

The effect of LS intake on serum lipids is shown in Table 8. No significant changes occurred in the levels of total lipid, total cholesterol, phospholipid, triglycerides, lipoprotein and HDL-cholesterol throughout the experimental period. Low-density lipoprotein (LDL) and VLDL were increased on day 14 in the LS period, in particularly, significantly $(p<0.05)$ in fasting VLDL, but in five volunteers, decreased slightly from $440.0 \pm 79.8$ (mean $\pm \mathrm{SD}) \mathrm{mg} / \mathrm{dl}$ in fasting $(433.0 \pm 92.6$ 
Table 7. Effect of lactosucrose intake on fecal enzyme activities of 8 human volunteers

\begin{tabular}{lcccr}
\hline \multirow{2}{*}{ Enzyme } & Before intake & \multicolumn{2}{c}{ During intake } & \multicolumn{1}{c}{$\begin{array}{c}\text { After intake } \\
\text { Day } 14\end{array}$} \\
\cline { 3 - 4 } & Day 0 & Day 7 & Day 14 & \\
\hline$\beta$-Glucuronidase & $13.25 \pm 5.31 a$ & $8.11 \pm 6.54$ & $5.96 \pm 4.29 *$ & $21.57 \pm 15.68$ \\
Nitroreductase & $2.64 \pm 0.43$ & $1.80 \pm 0.89$ & $1.95 \pm 0.70$ & $2.11 \pm 0.49$ \\
Azoreductase & $1.25 \pm 0.79$ & $0.79 \pm 0.61$ & $0.70 \pm 0.23$ & $1.02 \pm 0.39$ \\
$\beta$-Glucosidase & $43.48 \pm 30.52$ & $38.96 \pm 33.62$ & $16.89 \pm 11.22$ & $38.98 \pm 33.45$ \\
\hline
\end{tabular}

a Data expressed as mean $\pm \mathrm{SD}$ of $\mu \mathrm{mol} / \mathrm{h} / \mathrm{g}$ wet feces.

* Significant difference $(p<0.05)$ from the values of day 0 (before the intake).

Table 8. Effect of lactosucrose intake on serum lipid of 8 human volunteers

\begin{tabular}{lccr}
\hline Lipid & Before intake & During intake & After intake \\
& Day 0 & Day 14 & Day 14 \\
\hline Total lipids (mg/dl) & $560.3 \pm 73.8^{b}$ & $560.9 \pm 82.3$ & $558.0 \pm 109.9$ \\
& $561.3 \pm 72.8$ & $566.0 \pm 70.4$ & $584.1 \pm 132.4$ \\
Total cholesterol (mg/dl) & $177.3 \pm 24.2$ & $173.0 \pm 24.4$ & $174.7 \pm 30.5$ \\
& $173.8 \pm 26.1$ & $167.4 \pm 22.9$ & $171.1 \pm 29.3$ \\
Triglycerides (mg/dl) & $95.0 \pm 26.3$ & $105.8 \pm 36.3$ & $95.0 \pm 40.7$ \\
& $103.1 \pm 46.3$ & $121.7 \pm 36.8$ & $125.0 \pm 59.5$ \\
Phospholipids (mg/dl) & $198.7 \pm 18.1$ & $194.9 \pm 22.9$ & $200.3 \pm 26.4$ \\
& $196.9 \pm 20.0$ & $192.6 \pm 20.1$ & $201.7 \pm 32.1$ \\
$\alpha$-Lipoprotein (\%) & $34.8 \pm 2.5$ & $36.0 \pm 3.0$ & $37.5 \pm 4.7$ \\
& $33.9 \pm 3.4$ & $33.7 \pm 3.3$ & $33.7 \pm 4.5$ \\
Pre $\beta$-Lipoprotein (\%) & $22.2 \pm 3.3$ & $22.8 \pm 4.0$ & $21.0 \pm 4.0$ \\
$\beta$-Lipoprotein (\%) & $20.9 \pm 4.1$ & $23.2 \pm 4.0$ & $24.2 \pm 3.7$ \\
& $39.9 \pm 3.3$ & $39.8 \pm 3.5$ & $40.6 \pm 3.4$ \\
HDL-cholesterol (mg/dl) & $40.3 \pm 5.8$ & $38.0 \pm 3.8$ & $38.6 \pm 2.7$ \\
& $46.0 \pm 8.6$ & $44.1 \pm 7.9$ & $44.9 \pm 8.3$ \\
LDL $a$ (mg/dl) & $44.3 \pm 8.5$ & $43.6 \pm 6.7$ & $43.0 \pm 8.8$ \\
& $423.9 \pm 73.3$ & $450.1 \pm 77.1$ & $439.4 \pm 83.3$ \\
VLDL $a$ (mg/dl) & $412.1 \pm 86.8$ & $429.1 \pm 75.5$ & $423.9 \pm 76.5$ \\
& $126.7 \pm 47.7$ & $150.3 \pm 59.6 *$ & $125.8 \pm 56.9$ \\
& $115.6 \pm 30.7$ & $167.1 \pm 53.2$ & $162.4 \pm 78.0$
\end{tabular}

$a$ LDL: Low-density lipoprotein, VLDL: Very low-density lipoprotein.

$\checkmark$ Data are expressed as mean \pm SD. The upper and lower lines are the values of before and after meal, respectively.

Statistically significant at $*=p<0.05$ level when compared with the values before the intake.

Table 9. Effect of lactosucrose intake on fecal weight, $\mathrm{pH}$ and water content of 8 human volunteers

\begin{tabular}{|c|c|c|c|c|}
\hline \multirow{2}{*}{ Item } & \multirow{2}{*}{$\begin{array}{c}\text { Before intake } \\
\text { Day } 0\end{array}$} & \multicolumn{2}{|c|}{ During intake } & \multirow{2}{*}{$\begin{array}{l}\text { After intake } \\
\text { Day } 7\end{array}$} \\
\hline & & Day 7 & Day 14 & \\
\hline Fecal weight (g) & $95.6 \pm 16.2^{a}$ & $104.7 \pm 16.9$ & $108.0 \pm 17.9$ & $97.1 \pm 18.9$ \\
\hline $\mathrm{pH}$ & $6.3 \pm 0.3$ & $5.9 \pm 0.3 *$ & $5.8 \pm 0.4 *$ & $6.2 \pm 0.2$ \\
\hline Water content (\%) & $74.9 \pm 4.2$ & $78.4 \pm 5.2$ & $79.6 \pm 2.5 *$ & $75.9 \pm 2.7$ \\
\hline
\end{tabular}

a Data expressed as mean $\pm \mathrm{SD}$.

* Significant difference $(p<0.05)$ from the values of day 0 (before the intake). 
$\mathrm{mg} / \mathrm{dl}$ after meal) and $114.2 \pm 44.3 \mathrm{mg} / \mathrm{dl}(103.6 \pm 37.8 \mathrm{mg} / \mathrm{dl})$ before LS intake to $439.2 \pm 94.6 \mathrm{mg} / \mathrm{dl}$ in fasting $(430.4 \pm 83.8 \mathrm{mg} / \mathrm{dl}$ after meal $)$ and $107.4 \pm 28.6$ $\mathrm{mg} / \mathrm{dl}(102.0 \pm 33.2 \mathrm{mg} / \mathrm{dl})$ on day 14 of the intake, respectively.

\section{Fecal Properties}

Fecal water content was significantly $(p<0.05)$ increased on day 14 in LS period, whereas fecal $\mathrm{pH}$ values were significantly $(p<0.05)$ decreased during LS intake (Table 9). Fecal weight was increased at the rate of $9.5 \%$ and $13.0 \%$ on days 7 and 14 in the LS period, respectively, compared with before LS intake. The offensive odor of the feces was remarkably decreased during LS intake.

\section{DISCUSSION}

Although Fujita et al. (6) reported that LS was fermented by all species of Bifidobacterium, except for $B$. bifidum in vitro, our results showed that LS was fermented by all Bifidobacterium species including B. bifidum. The fermentation of LS by the intestinal bacteria was similar to that of lactulose (9).

LS escapes digestion by intestinal enzymes. Thus, it is not absorbed in the small intestine, and passes directly into the colon where it is fermented by LS-fermenting bacteria such as bifidobacteria which produces short chain fatty acids. The significant increase observed in bifidobacteria during LS intake (3 g or $6 \mathrm{~g}$ /day) was similar to those during intake of various oligosaccharides [lactulose $3 \mathrm{~g}$ (29), xylooligosaccharides $5 \mathrm{~g}$ (22), FOS $8 \mathrm{~g}$ (9), 4'-galactosyllactose $8 \mathrm{~g} \mathrm{(20),}$ galacto-oligosaccharides $10 \mathrm{~g}(12)$, transgalactosyl oligosaccharides (TOS) $10 \mathrm{~g}$

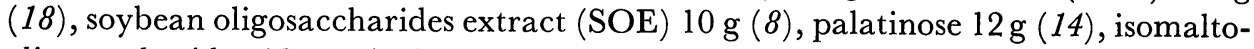
oligosaccharides $13.5 \mathrm{~g}$ (15), raffinose $15 \mathrm{~g}$ (2), or lactulose $30 \mathrm{~g}$ (11) per day] for healthy adults.

The significant decrease observed in $C$. perfringens, lecithinase-negative clostridia and bacteroidaceae during LS intake was similar to those during intake of raffinose (2), TOS (28), and lactulose (29), and suggests that an increase in fermentative anaerobes inhibits the growth of proteolytic bacteria during lactulose administration as reported by Vince et al. (33).

A decrease in clostridia has not been observed during intake of other oligosaccharides in previous reports $(8,9,12,14,15,20,22)$. The discrepancies between our data and hitherto reported results may be due to heat treatment to detect lecithinase-positive clostridia and the use of Medium 10 for lecithinase-negative clostridia in our study, because the detection rate of clostridia (lecithinase-positive and lecithinase-negative) was markedly increased when these methods were used (unpublished data).

The tendency for enterobacteriaceae to decrease by day 14 during LS intake was similar to that of lactulose intake (29) but not to that of TOS (3 g/day) (28). The significant decrease in bacteroidaceae during LS intake showed a negative correlation with the increase in the number of fecal bifidobacteria and contributed to the decrease in total bacteria.

The putrefactive products in the human intestine are mainly composed of 
potentially toxic substances $(3,33)$ such as ammonia, sulfide, indole and phenols produced from amino acids and urea. The significant decrease of fecal ammonia concentration despite an increase in fecal weights in this study due to LS fermentation may lead to inhibition of deaminase and urease activity by decreasing the $\mathrm{pH}$ and increasing short chain fatty acids (7) in the large intestine. The decreased fecal sulfide concentration during LS intake showed a positive correlation with the decrease in $C$. perfringens and bacteroidaceae which produce hydrogen sulfide (19).

The enzyme activities of $\beta$-glucuronidase, nitroreductase, azoreductase, and $\beta$-glucosidase have been shown to be associated with a wide variety of intestinal bacteria (1). The decrease in fecal $\beta$-glucuronidase activity during LS intake was the same as the results of polydextrose (4) and lactulose (29) intake, and may be be a result of the decreased activity in $C$. perfringens and bacteroidaceae.

It has been reported that intake of dietary fiber leads to a decrease in serum lipids, blood sugar, blood pressure, and body weight. Hidaka et al. $(9,10)$ demonstrated that FOS intake reduced serum triglycerides and free fatty acids in patients with hyperlipidemia. They (10) also reported a tendency for serum total cholesterol and apoprotein A to decrease after FOS intake for 1 month in patients with hypercholesterolemia. The reason for the increase in serum VLDL on day 14 during LS intake was not clear. It may be that an increase in apolipoprotein during LS intake results from the absorption of LS metabolites that affect hepatic function and are synthesized into apolipoprotein in the liver.

Ogata et al. (21) reported that the minimum effective dose of LS was 1-2 g/day for an increase of bifidobacteria, a decrease in clostridia, ammonia and sulfide in the feces. The results in present study showed that $3 \mathrm{~g} \mathrm{LS} /$ day is sufficient effective dose for an increase in short chain fatty acids, a decrease in indole and phenol, and a significant lowering of $\mathrm{pH}$ for all healthy adults. Furthermore, although the intake of $3 \mathrm{~g} \mathrm{LS} /$ day was associated with decreased $\beta$-glucuronidase and increased water content in the healthy adults, the difference was not significant (unlike $6 \mathrm{~g}$ LS/day intake). From these results, it was suggested that the effective dose of LS for healthy adults is $3 \mathrm{~g} /$ day.

\section{REFERENCES}

(1) Adlercreutz, H., and F. Martin. 1980. Biliary excretion and intestinal metabolism of progesterone and estrogens in man. J. Steroid Biochem. 13: 231-244.

(2) Benno, Y., K. Endo, N. Shiragami, K. Sayama, and T. Mitsuoka. 1987. Effect of raffinose on human fecal microflora. Bifidobacteria Microflora 6: 59-63.

(3) Bone, E., A. Tamm, and M. Hill. 1976. The production of urinary phenols by gut bacteria and their possible role in the causation of large bowel cancer. Am. J. Clin. Nutr. 29: 1448-1454.

(4) Endo, K., M. Kumemura, K. Nakamura, T. Fujisawa, K. Suzuki, Y. Benno, and T. Mitsuoka. 1991. Effect of high cholesterol diet and polydextrose supplementation on the microflora, bacterial enzyme activity, putrefactive products, volatile fatty acid (VFA) profile, weight, and $\mathrm{pH}$ of the feces in healthy volunteers. Bifidobacteria Microflora 10: 63-64.

(5) Fujita, K., K. Hara, H. Hashimoto, and S. Kitahata. 1990. Transfructosylation catalyzed by $\beta$-fructofuranosidase I from Arthrobacter sp. K-1. Agric. Biol. Chem. 54: 2655-2661.

(6) Fujita, K., K. Hara, S. Sakai, T. Miyae, M. Yamasita, Y. Tsunetomi, and T. Mitsuoka. 1991. 
Effect of lactosucrose on intestinal flora and its digestibility. J. Jpn. Soc. Starch Sci. 38: 249-255 (in Japanese).

(7) Hawksworth, G.M., B.S. Drasar, and M.J. Hill. 1971. Intestinal bacteria and the hydrolysis of glycosidic bonds. J. Med. Microbiol. 4: 451-459.

(8) Hayakawa, K., T. Mizutani, K. Wada, T. Masai, I. Yoshihara, and T. Mitsuoka. 1990. Effects of soybean oligosaccharides on human faecal flora. Microbial. Ecol. Health Dis. 3: 293-303.

(9) Hidaka, H., T. Eida, T. Takizawa, T. Tokunaga, and Y. Tashiro. 1986. Effects of fructooligosaccharides on intestinal flora and human health. Bifidobacteria Microflora 5: 37-50.

(10) Hidaka, H., Y. Tashiro, and T. Eida. 1991. Proliferation of bifidobacteria by oligosaccharides and their useful effect on human health. Bifidobacteria Microflora 10: 65-79.

(11) Hoffmann, K., and J. Bricher. 1969. Veränderungen der bakteriellen Darmbesiedelung nach Laktulosegaben. Schweiz. Med. Woch. 99: 608-609.

(12) Ito, M., Y. Deguchi, A. Miyamori, K. Matsumoto, H. Kikuchi, K. Matsumoto, Y. Kobayashi, T. Yajima and T. Kan. 1990. Effects of administration of galacto-oligosaccharides on the human faecal microflora, stool weight and abdominal sensation. Microbial Ecol. Health Dis. 3: 285292.

(13) Kasezawa, N., and H. Murakami. 1983. Serum total and free cholesterol values and ratios of cholesterol ester in various physiological factors of healthy adult subjects by enzymatic analysis. Igakuno Ayumi 126: 646-652 (in Japanese).

(14) Kashimura, J., Y. Nakajima, Y. Benno, K. Endo, and T. Mitsuoka. 1989. Effects of palatinose and its condensate intake on human fecal microflora. Bifidobacteria Microflora 8: 45-50.

(15) Kohmoto, T., F. Fukui, H. Takaku, Y. Machida, M. Arai, and T. Mitsuoka. 1988. Effect of isomalto-oligosaccharides on human fecal flora. Bifidobacteria Microflora 7: 61-69.

(16) Masuda, Y., K. Hirota, K. Yoneya, T. Kajikawa, H. Kushiro, K. Itoh, J. Kodama, and Y. Nakao. 1982. Automatic analysis for triglycerides with free glycerol blank subtraction. Anal. Clin. Specimen. 51 : 87-91.

(17) Mitsuoka, T. 1990. Bifidobacteria and their role in human health. J. Indust. Microbiol. 6: 263-268.

(18) Mitsuoka, T., K. Ohno, Y. Benno, K. Suzuki, und K. Nanba. 1976. Die Faekalflora bei Menshen. Mitteilung: Vergleich des neu entwickelten Verfahrens mit den bisherigen üblichen Verfahren zur Darmfloraanalyse. Zentralbl. Bakteriol. Parasitenkd. Infectionskr. Hyg., I. Abt. Orig. A234: 219-233.

(19) Morishita, Y. 1990. Chonaifurora no kozo to kinou. pp. 122. Asakura Shoten Co., Ltd., Tokyo (in Japanese).

(20) Ohtsuka, K., Y. Benno, K. Endo, H. Ueda, O. Ozawa, Y. Uchida, and T. Mitsuoka. 1989. Effects of 4' galactosyllactose intake on human fecal microflora. Bifidus 2: 143-149 (in Japanese).

(21) Ogata, Y., K. Fujita, H. Ishigami, K. Hara, A. Terada, H. Hara, I. Fujimori, and T. Mitsuoka. 1993. Effect of small amount of $4^{G_{-}} \beta$-D-galactosylsucrose (lactosucrose) on fecal flora and fecal properties. J Jpn. Soc. Nutr. Food Sci. 46: 317-323 (in Japanese).

(22) Okazaki, M., S. Fujikawa, and N. Matsumoto. 1990. Effect of xylooligosaccharide on the growth of bifidobacteria. Bifidobacteria Microflora 9: 77-86.

(23) Oshima, S., E. Nakano, and T. Tuchiya. 1976. Electrophoresis assay and immunoassay in lipoprotein. Rinsho Kensa 25: 129-134 (in Japanese).

(24) Rowland, I.R., A. Wise, and A.K. Mallet. 1983. Metabolic profile of caecal microorganisms from rats fed indigestible plant cell-wall component. Food Chem. Toxicol. 21: 25-29.

(25) Sasaki, T., K. Tanemura, and N. Kubota. 1977. Evaluation of the serum lipoprotein fraction values estimated by the turbidimetric method. Jpn. J. Clin. Pathol. 25: 931-934.

(26) Shimizu, S., K. Inoue, Y. Tani, and H. Yamane. 1979. Enzymatic microdetermination of serum free fatty acids. Anal. Biochem. 98: 341-345.

(27) Takayama, M., S. Itoh, T. Nagasaki, and I. Tanimizu. 1977. A new enzymatic method for determination of serum choline-containing phospholipids. Clin. Chim. Acta. 79: 93-98.

(28) Tanaka, R., H. Takayama, M. Morotomi, 'T. Kuroshima, S. Ueyama, K. Matsumoto, A. Kuroda, and M. Mutai. 1983. Effects of administration of TOS and Bifidobacterium breve 4006 on 
the human fecal flora. Bifidobacteria Microflora 2: 17-24.

(29) Terada, A., H. Hara, M. Kataoka, and T. Mitsuoka. 1992. Effect of lactulose on the composition and metabolic activity of the human faecal flora. Microbial Ecol. Health Dis. 5: 43-50.

(30) Terada, A., H. Hara, S. Kato, T. Kimura, I. Fujimori, K. Hara, T. Maruyama, and T. Mitsuoka. 1993. Effect of lactosucrose $\left(4^{\mathrm{G}}-\beta-\mathrm{D}-\mathrm{galactosylsucrose}\right)$ on fecal flora and fecal putrefactive products of cats. J. Vet. Med. Sci. 55: 291-295.

(31) Terada, A., H. Hara, T. Oishi, S. Matsui, T. Mitsuoka, S. Nakajyo, I. Fujimori, and K. Hara. 1992. Effect of dietary lactosucrose on faecal flora and faecal metabolites of dogs. Microbial Ecol. Health Dis. 5: 87-92.

(32) Terada, A., H. Hara, A. Hemmi, M. Kataoka, and T. Mitsuoka. 1993. A simple and rapid method for the determination of excretal ammonia using ammonia gas sensing electrode. Anim. Sci. Technol. 64: 719-722.

(33) Vince, A., P.F. Down, J. Murison, F.J. Twigg, and O.M. Wrong. 1976. Generation of ammonia. from non-urea sources in a fecal incubation system. Clin. Sci. Molec. Med. 51: 313-322.

(34) Yoneyama, M., T. Mandai, H. Ago, K. Fujii, and Y. Katayama. 1992. Effects of $4^{G}$ - $\beta$-D-galactosylsucrose (lactosucrose) intake on intestinal flora in healthy humans. J. Jpn. Soc. Nutr. Food Sci. 45: 101-107 (in Japanese).

(35) Yoshihara, I. 1981. Isothermal gas chromatographic analysis of putrefactive products in gastrointestinal contents and urine using the same dual column system. Agric. Biol. Chem. 45: 18731875. 